\author{
United Nations Educational Scientific and Cultural Organization \\ and \\ International Atomic Energy Agency \\ INTERNATIONAL CENTRE FOR THEORETICAL PHYSICS
}

\title{
NON-COMMUTATIVE CHERN CHARACTERS OF COMPACT LIE GROUP C*-ALGEBRAS
}

\author{
Do Ngoc Diep \\ Institute of Mathematics, National Centre for Natural Science and Technology, \\ P.O. Box 631, Bo Ho, 10.000, Hanoi, Vietnam, 巴 \\ Aderemi O. Kuku \\ International Centre for Theoretical Physics, \\ ICTP P. O. Box 586, 34100, Trieste, Italy 9 \\ and \\ Nguyen Quoc Tho \\ Department of Mathematics, Vinh University, Vinh City, Vietnam, \\ and $\mathrm{c} / \mathrm{o}$ : \\ Institute of Mathematics, National Centre for Natural Science and Technology, \\ P.O. Box 631, Bo Ho, 10.000, Hanoi, Vietnam. ${ }^{[3}$
}

\begin{abstract}
For compact Lie groups the Chern characters $c h: K^{*}(G) \otimes \mathbf{Q} \rightarrow H_{D R}^{*}(G ; \mathbf{Q})$ were already constructed. In this paper we propose the corresponding non-commutative Chern characters, which are also homomorphisms from quantum $K$-groups into entire current periodic cyclic homology groups of group $\mathrm{C}^{*}$-algebras $c h_{C^{*}}: K_{*}\left(C^{*}(G)\right) \rightarrow H E_{*}\left(C^{*}(G)\right)$. We obtain also the corresponding algebraic version $c h_{\text {alg }}: K_{*}\left(C^{*}(G)\right) \rightarrow H P_{*}\left(C^{*}(G)\right)$, which coincides with the Fedosov-Cuntz-Quillen formula for Chern characters.
\end{abstract}

MIRAMARE - TRIESTE

November 1997

\footnotetext{
${ }^{1}$ E-mail: dndiep@thevinh.ncst.ac.vn

${ }^{2}$ E-mail: kuku@ictp.trieste.it

${ }^{3}$ E-mail: dndiep@thevinh.ncst.ac.vn
} 


\section{INTRODUCTION}

Let $G$ be a compact Lie group, $H_{D R}^{*}(G ; \mathbf{Q})$ the rational de Rham cohomology. It is classical that the Chern character

$$
c h: K^{*}(G) \otimes \mathbf{Q} \rightarrow H_{D R}^{*}(G ; \mathbf{Q})
$$

is an isomorphism, see [16]. There have also been other Chern characters from K-theory to such (co)homologies as Hochschild and cyclic (co)homologies, etc and the theories have played important role in geometry, index theory etc. More recently, some of the theories e.g. cyclic cohomology, have been quantized as the so called non-commutative geometry. Since then, there has been more need to define non-commutative version of Chern characters that would play in quantum theories similar roles to the ordinary commutative Chern characters in classical theories. The aim of this paper is to construct such noncommutative Chern characters. In $\S 2$ we review some preliminaries on $C^{*}(G)$ for compact Lie groups $G$ and $K K$ functors. We also define the periodic cyclic homology $\operatorname{HP}_{*}(\mathrm{~A})$ and entire cyclic homology $\mathrm{HE}_{*}(\mathrm{~A})$ for a general involutive Banach algebra $A$.

We define an entire cyclic homology $\operatorname{HE}_{*}(\mathrm{~A})$ of an involutive Banach algebra $A$ via a family of ideals with ad-invariant trace and via projective and inductive topologies to form non-commutative analogues of de Rham currents. We use the dual to the entire cochain of A. Connes at the level of ideals with ad-invariant trace. When $A=C^{*}(G)$, where $G$ is a compact Lie group, our theory coincides with the usual de Rham cohomology yielding the calculation in the work of Watanabe. (See [16], [17]). Some of these calculations are briefly reviewed in 3.3 .

In $\S 3$, we define a non-commutative Chern character $c h: K_{*}(A) \rightarrow \mathrm{HE}_{*}(\mathrm{~A})$ (see 3.1) and show in 3.2 that when $A=C^{*}(G)$ for a compact Lie group $G$, ch is an isomorphism, which can be identified with the classical Chern character $K_{*}^{W}(\mathbf{C}(\mathbf{T})) \rightarrow \mathrm{HE}_{*}^{\mathrm{W}}(\mathbf{C}(\mathbf{T}))$ that is also an isomorphism where $\mathbf{T}$ is a maximal torus of $G$ with Weyl group $W$. In $\S 4$, we indicate at first that the periodic cyclic homology $\mathrm{HP}_{*}(\mathrm{~A})$ defined in $\S 2$ through ideals of $A$ coincides with the periodic cyclic homology of Cuntz-Quillen [6] when $A=C^{*}(G)$ for a compact Lie group $G$. We then go on to show in 4.5 that the Chern character ch $: K_{*}\left(C^{*}(G)\right) \rightarrow \mathrm{HP}_{*}\left(\mathrm{C}^{*}(\mathrm{G})\right)$ is an isomorphism which can be identified with the classical Chern character $K_{*}(\mathbf{C}(\mathbf{T})) \rightarrow \mathrm{HP}_{*}(\mathbf{C}(\mathbf{T}))$ which is also an isomorphism.

Notes on Notation: For any compact Lie group $G$, we write $K^{*}(G)$ for the $\mathbf{Z} / 2$ graded topological K-theory of $G$. We use Swan's theorem to identify $K^{*}(G)$ with the $\mathbf{Z} / 2$-graded $K_{*}(C(G))$. For any involutive Banach algebra $A, K_{*}(A), \mathrm{HE}_{*}(\mathrm{~A}), \mathrm{HP}_{*}(\mathrm{~A})$, are $\mathbf{Z} / 2$-graded algebraic K-group of $A$, entire cyclic homology of $A$, and periodic cyclic homology of $A$, respectively. If $\mathbf{T}$ is a maximal torus of $G$ with the Weyl group $W$, write $\mathbf{C}(\mathbf{T})$ for the ring of complex valued functions on $\mathbf{T}$. We write $K_{*}^{W}(\mathbf{C}(\mathbf{T}))$, (resp. $\mathrm{HE}_{*}^{\mathrm{W}}(\mathbf{C}(\mathbf{T}))$ for the $\mathbf{Z} / 2$-graded $W$-equivariant K-group, (resp. entire cyclic homology) of $\mathbf{C}(\mathbf{T})$. 


\section{Preliminaries}

2.1. Group $\mathbf{C}^{*}$-Algebras. We introduce in this subsection the definition and the main properties of $\mathrm{C}^{*}$-algebras of compact Lie groups we need for later use. Let us recall that in a compact Lie group $G$ there exists a (bi)invariant Haar measure denoted $d g$. Consider the involutive Banach algebra $L^{1}(G)$ of absolutely integrable complex-valued functions on $G$ with the convolution product

$$
(f * g)(x):=\int_{G} f(y) g\left(y^{-1} x\right) d y,
$$

involution

$$
f^{*}(x):=\overline{f\left(x^{-1}\right)}
$$

and the $L^{1}$-norm

$$
\|f\|:=\int_{G}|f(x)| d x .
$$

This algebra is not regular in the sense that in general

$$
\|\varphi * \psi\| \neq\|\varphi\|\|\psi\| \text {. }
$$

Let us consider the new regular norm defined by

$$
\|\varphi\|:=\sup _{\pi \in \hat{G}}\|\pi(\varphi)\|,
$$

where by definition, $\hat{G}$ is the dual object of $G$, i.e. the set of (unitary) equivalence classes of irreducible (unitary) representations of $G$. The completion of the involutive Banach algebra $L^{1}(G)$ with respect to this norm is a regular complete involutive Banach algebra with unit element, which is called the group $C^{*}$-algebra of $G$.

The following facts are well known, see [13.

1. The dual object $\hat{G}$ is not more than denumerable, finite if the group $G$ is finite and of cardinal $\mathbf{N}$ if $G$ is compact.

2. Every irreducible representation of the compact Lie group $G$ is equivalent to a unitary one (unitarization) and is finite dimensional, of dimension $n_{i}$, say

3. Every representation $\pi$ of $G$ can be extended to a *-representation of $C^{*}(G)$ by the Fourier-Gel'fand transform

$$
\hat{f}(\pi)=\pi(f):=\int_{G} \pi(x) f(x) d x .
$$

4. There is one-to-one correspondence between the irreducible representations $\pi$ of $G$ and the non-degenerate irreducible *-representation of $C^{*}(G)$.

5. Let us fix a bijection between $\pi_{n} \in \hat{G}$ and $n \in \mathbf{N}$. Then, there exists a constant $c=c_{f}$, where $f$ is in $C^{*}(G)$, such that

$$
\left\|\hat{f}(n)-c_{f} \cdot \operatorname{Id}\right\| \rightarrow 0,
$$

as $n \rightarrow \infty$. 
Let us denote the restricted Cartesian product of matrix algebras $\operatorname{Mat}_{\mathrm{n}_{\mathrm{i}}}(\mathbf{C})$ by

$$
\prod_{i=1}^{\prime} \operatorname{Mat}_{\mathrm{n}_{\mathrm{i}}}(\mathbf{C})=\left\{\hat{\mathrm{f}} \quad ; \quad\left\|\hat{\mathrm{f}}(\mathrm{n})-\mathrm{c}_{\mathrm{f}} . \mathrm{Id}\right\| \rightarrow 0, \text { as } \mathrm{n} \rightarrow \infty\right\}
$$

The main property of the group $\mathrm{C}^{*}$-algebra $C^{*}(G)$ is the fact that

$$
C^{*}(G) \cong \prod_{i=1}^{\prime} \operatorname{Mat}_{\mathrm{n}_{\mathrm{i}}}(\mathbf{C})
$$

This means also that

$$
C^{*}(G)=\lim _{\mathrm{N}} I_{N}
$$

and that the right-hand side is dense in norm, where

$$
I_{N}:=\prod_{i=1}^{N} \operatorname{Mat}_{\mathrm{n}_{\mathrm{i}}}(\mathbf{C})
$$

are two-sided closed ideals in $C^{*}(G)$.

2.2. KK-groups. We recall in this subsection a brief definition of operator K-functors. Following G. G. Kasparov, a Fredholm representation of a $\mathrm{C}^{*}$-algebra $A$ is a triple $\left(\pi_{1}, \pi_{2}, F\right)$, consisting of ${ }^{*}$-representations $\pi_{1}, \pi_{2}: A \rightarrow \mathcal{L}\left(\mathcal{H}_{B}\right)$ and a Fredholm operator $F \in \mathcal{F}\left(\mathcal{H}_{B}\right)$, admitting an adjoint operator, on the Hilbert $\mathrm{C}^{*}$-module $\mathcal{H}_{B}=\ell_{B}^{2}$ over $\mathrm{C}^{*}$-algebra $B$, satisfying the relations

$$
\pi_{1}(a) F-F \pi_{2}(a) \in \mathcal{K}_{B}
$$

where $\mathcal{K}_{B}$ is the ideal of compact (adjointable) $\mathrm{C}^{*}$-module endomorphisms of $\mathcal{H}_{B}$. The classes of homotopy invariance and unitary equivalence of Fredholm modules form the so called Kasparov operator K-group $K K^{*}(A, B)$. Herewith put $A=C^{*}(G), B=\mathbf{C}$, where $G$ is a compact Lie group. In this case we have $K_{*}\left(C^{*}(G)\right)=K K^{*}\left(C^{*}(G), \mathbf{C}\right)$, where $K_{*}(A)$ is algebraic $K$-group of $A$. Note that

$$
\begin{aligned}
K K_{0}(A, \mathbf{C}) \cong K_{0}(A), \\
K K_{1}(A, \mathbf{C}) \cong K_{1}(A) .
\end{aligned}
$$

2.3. Entire Homology of Involutive Banach Algebras. Let $A$ be an involutive Banach algebra. Recall that $\mathrm{A}$. Connes defines entire cyclic cohomology $\operatorname{HE}^{*}(\mathrm{~A})$ and a pairing

$$
K_{*}(A) \times \mathrm{HE}^{*}(\mathrm{~A}) \rightarrow \mathbf{C} .
$$

Also M. Khalkhali [1] proved Morita and homotopy invariance of $\operatorname{HE}^{*}(\mathrm{~A})$. We now define the entire homology $\mathrm{HE}_{*}(\mathrm{~A})$ as follows: Given a collection $\left\{I_{\alpha}\right\}_{\alpha \in \Gamma}$ of ideals in $A$, equipped with a so called $\mathrm{ad}_{\mathrm{A}}$-invariant trace

$$
\tau_{\alpha}: I_{\alpha} \rightarrow \mathbf{C}
$$

satisfying the properties:

1. $\tau_{\alpha}$ is a continuous linear functional, normalized as $\left\|\tau_{\alpha}\right\|=1$, 
2. $\tau_{\alpha}$ is positive in the sense that

$$
\tau_{\alpha}\left(a^{*} a\right) \geq 0, \forall \alpha \in \Gamma
$$

where the map $a \mapsto a^{*}$ is the involution defining the involutive Banach algebra structure, i.e. an anti-hermitian endomorphism such that $a^{* *}=a$

3. $\tau_{\alpha}$ is strictly positive in the sense that $\tau_{\alpha}\left(a^{*} a\right)=0$, iff $a=0$, for every $\alpha \in \Gamma$.

4. $\tau_{\alpha}$ is $\operatorname{ad}_{\mathrm{A}}$-invariant in the sense that

$$
\tau_{\alpha}(x a)=\tau_{\alpha}(a x), \forall x \in A, a \in I_{\alpha},
$$

then we have for every $\alpha \in \Gamma$ a scalar product

$$
\langle a, b\rangle_{\alpha}:=\tau_{\alpha}\left(a^{*} b\right)
$$

and also an inverse system $\left\{I_{\alpha}, \tau_{\alpha}\right\}_{\alpha \in \Gamma}$. Let $\bar{I}_{\alpha}$ be the completion of $I_{\alpha}$ under the scalar product above and $\widetilde{\bar{I}_{\alpha}}$ denote $I_{\alpha}$ with formally adjoined unity element. Define the $C^{n}\left(\widetilde{\bar{I}_{\alpha}}\right)$ the set of $n+1$-linear maps $\varphi:\left(\widetilde{\bar{I}}_{\alpha}\right)^{n+1} \rightarrow \mathbf{C}$.

For $\alpha \leq \beta$, we have a well-defined map

$$
D_{\alpha}^{\beta}: C^{n}\left(\widetilde{\bar{I}_{\alpha}}\right) \rightarrow C^{n}\left(\widetilde{\bar{I}_{\beta}}\right),
$$

which makes $\left\{C^{n}\left(\widetilde{\bar{I}}_{\alpha}\right)\right\}$ into a direct system. Write $Q=\underset{\lim }{\longrightarrow} C^{n}\left(\widetilde{\bar{I}}_{\alpha}\right)$. Remark that it admits a Hilbert space structure, see [7]-[8]. Let $C_{n}(A):=\operatorname{Hom}\left(\underline{\lim } \mathrm{C}^{\mathrm{n}}\left(\widetilde{\overline{\mathrm{I}}}_{\alpha}\right), \mathbf{C}\right)$.

Let

$$
\begin{gathered}
b, b^{\prime}: C^{n}\left(\widetilde{\bar{I}}_{\alpha}\right) \rightarrow C^{n+1}\left(\widetilde{\bar{I}}_{\alpha}\right), \\
N: C^{n}\left(\widetilde{\bar{I}}_{\alpha}\right) \rightarrow C^{n}\left(\widetilde{\bar{I}}_{\alpha}\right), \\
\lambda: C^{n}\left(\widetilde{\bar{I}}_{\alpha}\right) \rightarrow C^{n}\left(\widetilde{\bar{I}}_{\alpha}\right), \\
S: C^{n+1}\left(\widetilde{\bar{I}}_{\alpha}\right) \rightarrow C^{n}\left(\widetilde{\bar{I}}_{\alpha}\right)
\end{gathered}
$$

be defined as in A. Connes [3]. We follow the notations in [1] . Denotes by $b^{*},\left(b^{\prime}\right)^{*}, N^{*}, \lambda^{*}, S^{*}$ the corresponding adjoint operators.

We now have a bi-complex

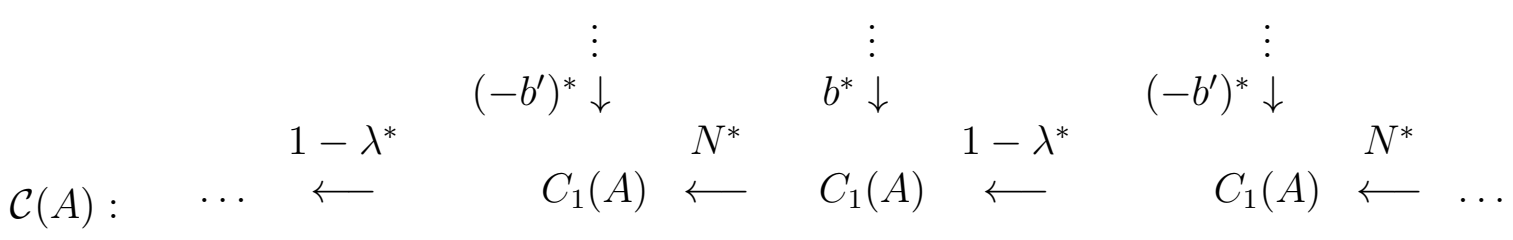

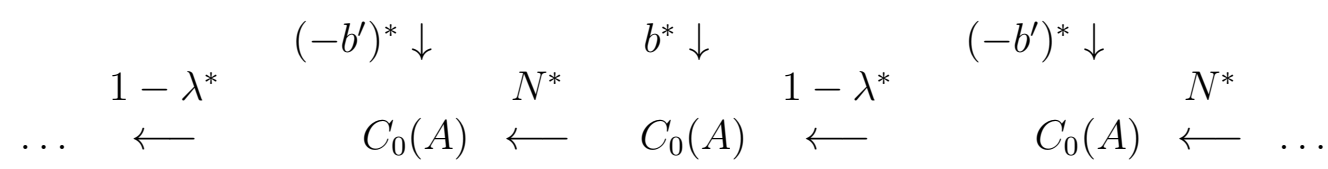

with $b^{*}$ in the even columns and $\left(-b^{\prime}\right)^{*}$ in the odd columns, where ${ }^{*}$ means the corresponding adjoint operator. Now we have

$$
\operatorname{Tot}\left(\mathcal{C}(\mathrm{A})^{\text {even }}\right)=\operatorname{Tot}\left(\mathcal{C}(\mathrm{A})^{\text {odd }}\right):=\oplus_{\mathrm{n} \geq 0} \mathrm{C}_{\mathrm{n}}(\mathrm{A}),
$$


which is periodic with period two. Hence, we have

$$
\bigoplus_{n \geq 0} C_{n}(A) \stackrel{\partial}{\longleftarrow} \bigoplus_{n \geq 0} C_{n}(A)
$$

where $\partial=d_{v}+d_{h}$ is the total differential.

Let $\mathrm{HP}_{*}(\mathrm{~A})$ be the homology of the total complex $(\operatorname{Tot} \mathcal{C}(\mathrm{A}))$. Remark that this $\mathrm{HP}_{*}(\mathrm{~A})$ is, in general different from the $\mathrm{HP}_{*}(\mathrm{~A})$ of Cuntz-Quillen.

Definition 2.1. An even (or odd) chain $\left(f_{n}\right)_{n \geq 0}$ in $\mathcal{C}(A)$ is called entire if the radius of convergence of the power series $\sum_{n} \frac{n !}{\left[\frac{n}{2}\right] !}\left\|f_{n}\right\| z^{n}, z \in \mathbf{C}$ is infinite.

Let $C_{e}(A)$ be the subcomplex of $C(A)$ consisting of entire chains. Then we have a periodic complex.

Theorem 2.2. Let

$$
\operatorname{Tot}\left(\mathrm{C}_{\mathrm{e}}(\mathrm{A})^{\mathrm{even}}\right)=\operatorname{Tot}\left(\mathrm{C}_{\mathrm{e}}(\mathrm{A})^{\text {odd }}\right):=\bigoplus_{\mathrm{n} \geq 0} \mathrm{C}_{\mathrm{n}}^{\mathrm{e}}(\mathrm{A})
$$

where $C_{n}^{e}(A)$ is the entire $n$-chain. Then we have a complex of entire chains with the total differential $\partial$

$$
\bigoplus_{n \geq 0} C_{n}^{e}(A) \underset{\partial}{\longleftarrow} \bigoplus_{n \geq 0} C_{n}^{e}(A)
$$

The homology of this complex is called also the entire homology and denoted by $\mathrm{HE}_{*}(\mathrm{~A})$. Note that this entire homology is defined through the inductive limits of ideals with adinvariance trace.

\section{Non-commutative Chern Characters for Involutive Banach Algebras}

Let $A$ be an involutive Banach algebra. In this section, we construct a non-commutative character

$$
c h_{C^{*}}: K_{*}(A) \rightarrow \mathrm{HE}_{*}(\mathrm{~A})
$$

and later show that when $A=C^{*}(G)$, this Chern character reduces up to isomorphism to classical Chern character.

Let $A$ be an involutive Banach algebra with unity.

Theorem 3.1. There exists a Chern character

$$
c h_{C^{*}}: K_{*}(A) \rightarrow \mathrm{HE}_{*}(\mathrm{~A}) .
$$

Proof. We first recall that there exists a pairing

$$
K_{n}(A) \times C^{n}(A) \rightarrow \mathbf{C}
$$


due to A. Connes, see [4]. Hence there exists a map $K_{n}(A) \stackrel{C_{n}}{\longrightarrow} \operatorname{Hom}\left(\mathrm{C}^{\mathrm{n}}(\mathrm{A}), \mathbf{C}\right)$. So, by 1.1 , we have for each $\alpha \in \mathbf{C}$, a map $K_{n}(A) \stackrel{C_{n}^{\alpha}}{\longrightarrow} \operatorname{Hom}\left(\mathrm{C}^{\mathrm{n}}\left(\widetilde{\overline{\mathrm{I}}_{\alpha}}\right), \mathbf{C}\right)$ and hence a map $K_{n}(A) \stackrel{C_{n}}{\longrightarrow} \operatorname{Hom}\left(\lim _{\alpha} \mathrm{C}^{\mathrm{n}}\left(\widetilde{\overline{\mathrm{I}}_{\alpha}}\right), \mathbf{C}\right)$. We now show that $C_{n}$ induces the Chern map

$$
c h: K_{n}(A) \rightarrow \mathrm{HE}_{\mathrm{n}}(\mathrm{A})
$$

Now let $e$ be an idempotent in $M_{k}(A)$ for some $k \in \mathbf{N}$. It suffices to show that for $n$ even, if $\varphi=\partial \psi$, where $\varphi \in C^{n}\left(\widetilde{\bar{I}_{\alpha}}\right)$ and $\psi \in C^{n+1}\left(\widetilde{\bar{I}_{\alpha}}\right)$, then

$$
\langle e, \varphi\rangle=\sum_{n=1}^{\infty} \frac{(-1)^{n}}{n !} \varphi(e, e, \ldots, e)=0 .
$$

However, this follows from Connes' results in ( $\mid 4]$,Lemma 7).

The proof of the case for $n$ odd would also follow from 迎.

Our next result computes the Chern character in 2.1 for $A=C^{*}(G)$ by reducing it to the classical case.

Theorem 3.2. Let $\mathbf{T}$ be a fixed maximal torus of $G$ with Weyl group $W:=N_{G}(\mathbf{T}) / \mathbf{T}$. Then the Chern character

$$
c h_{C^{*}}: K_{*}\left(\mathbf{C}^{*}(G)\right) \rightarrow \operatorname{HE}_{*}\left(\mathrm{C}^{*}(\mathrm{G})\right)
$$

is an isomorphism, which can be identified with the classical Chern character

$$
c h: K_{*}^{W}(\mathbf{C}(\mathbf{T})) \rightarrow \mathrm{HE}_{*}^{\mathrm{W}}(\mathbf{C}(\mathbf{T}))
$$

that is also an isomorphism.

Proof. First observe that we have an isomorphism

$$
\begin{aligned}
& K_{*}\left(C^{*}(G)\right) \cong K_{*}\left(\prod_{i=1}^{\prime \infty} \operatorname{Mat}_{\mathrm{n}_{\mathrm{i}}}(\mathbf{C})=\lim _{\longrightarrow} \mathrm{K}_{*}\left(\prod_{\mathrm{i}=1}^{\mathrm{N}} \operatorname{Mat}_{\mathrm{n}_{\mathrm{i}}}(\mathbf{C})\right) \cong\right. \\
& \cong K_{*}\left(\quad \prod \quad \mathbf{C}_{\lambda}\right)=K_{*}^{W}(\mathbf{C}(\mathbf{T})) \text {. } \\
& \lambda=\text { highest weight } \bmod \mathrm{W}
\end{aligned}
$$

Next we have

$$
\begin{gathered}
\mathrm{HE}_{*}\left(\mathrm{C}^{*}(\mathrm{G})\right) \cong \mathrm{HE}_{*}\left(\underset{\mathrm{lim}}{\longrightarrow} \prod_{\mathrm{i}=1}^{\mathrm{N}} \operatorname{Mat}_{\mathrm{n}_{\mathrm{i}}}(\mathbf{C})\right)=\underset{\lim }{\longrightarrow} \mathrm{HE}_{*}\left(\prod_{\mathrm{i}=1}^{\mathrm{N}} \operatorname{Mat}_{\mathrm{n}_{\mathrm{i}}}(\mathbf{C})\right) \\
\cong \mathrm{HE}_{*}\left(\quad \prod_{\left.\mathbf{C}_{\lambda}\right)}=\mathrm{HE}_{*}^{\mathrm{W}}(\mathbf{C}(\mathbf{T})),\right.
\end{gathered}
$$

$\lambda=$ highest weight mod $\mathrm{W}$

where $\mathbf{C}_{\lambda}=\mathbf{C}$ are enumerated by the highest weights of the corresponding irreducible representations of $G$.

Because the irreducible representations of compact Lie groups are defined by their characters (highest weights), the above product is indexed by highest weights modulo the action of the Weyl group $W$.

Furthermore, we have from standard results in topology that

$$
K_{W}^{*}(\mathbf{T}) \cong K_{W}^{*}(B \mathbf{T}) .
$$


Now consider the commutative diagram

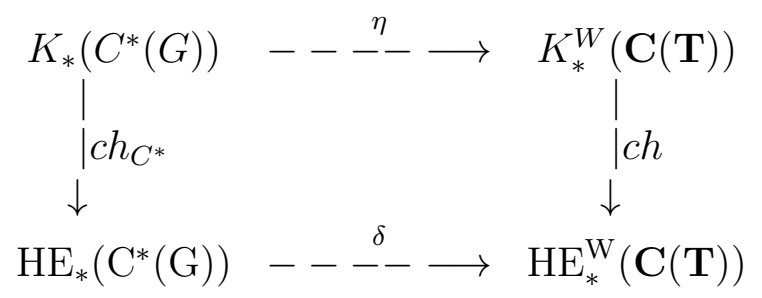

Since $\eta, \delta$ are isomorphisms and $c h$ is also an isomorphism, we have that $c h_{C^{*}}=\delta^{-1}(c h) \eta$ is also an isomorphism.

Remark 3.3. For some classical groups, e. g. $\mathrm{SU}(\mathrm{n}+1), \mathrm{SO}(2 \mathrm{n}+1), \mathrm{SU}(2 \mathrm{n}), S p(n)$ etc. the groups

$$
\begin{gathered}
K^{*}(G) \cong K_{*}^{W}(\mathbf{C}(\mathbf{T})) \cong K_{W}^{*}(\mathbf{T}) \cong K_{*}\left(C^{*}(G)\right) \cong \\
\cong \mathrm{HE}_{*}\left(\mathrm{C}^{*}(\mathrm{G})\right) \cong \mathrm{HE}_{*}^{\mathrm{W}}\left(\mathbf { C } ( \mathbf { T } ) \cong \mathrm { H } _ { * } ^ { \mathrm { W } } \left(\mathbf{C}(\mathbf{T}) \cong \mathrm{H}_{\mathrm{W}}^{*}(\mathbf{T}) \cong \mathrm{H}^{*}(\mathrm{G}) \cong \mathrm{HP}_{*}\left(\mathrm{C}^{*}(\mathrm{G})\right)\right.\right.
\end{gathered}
$$

are as follows.

(a). For any compact Lie group $G$, let $R[G]$ be the representation ring. Then the $\mathbf{Z} /(2)$-graded algebra $K^{*}(G)=\wedge_{\mathbf{C}}\left(\beta\left(\rho_{1}\right), \beta\left(\rho_{2}\right), \ldots, \beta\left(\rho_{n}\right)\right)$, where $\rho_{i}$ are the standard irreducible representations and $\beta: R[G] \rightarrow K^{*}(G)$ is the Bott map. Hence, from [16] we have

$$
\begin{gathered}
K^{*}(\mathrm{SU}(\mathrm{n}+1)) \cong \wedge_{\mathbf{C}}\left(\beta\left(\rho_{1}\right), \ldots, \beta\left(\rho_{\mathrm{n}}\right)\right), \\
K^{*}(\mathrm{SO}(2 \mathrm{n}+1)) \cong \wedge_{\mathbf{C}}\left(\beta\left(\rho_{1}\right), \ldots, \beta\left(\rho_{\mathrm{n}}\right), \varepsilon_{2 \mathrm{n}+1}\right) .
\end{gathered}
$$

(b). It follows from [17] that the $\mathbf{Z} /(2)$-graded complex cohomology groups are exterior algebras over $\mathbf{C}$ and in particular

$$
\begin{gathered}
H^{*}(\mathrm{SU}(2 \mathrm{n})) \cong \wedge_{\mathbf{C}}\left(\mathrm{x}_{3}, \mathrm{x}_{5}, \ldots, \mathrm{x}_{4 \mathrm{n}-1}\right), \\
H^{*}(\operatorname{Sp}(n)) \cong \wedge_{\mathbf{C}}\left(x_{3}, x_{7}, \ldots, x_{4 n-1}\right), \\
H^{*}(\mathrm{SU}(2 \mathrm{n}+1)) \cong \wedge_{\mathbf{C}}\left(\mathrm{x}_{3}, \mathrm{x}_{5}, \ldots, \mathrm{x}_{4 \mathrm{n}+1}\right), \\
H^{*}(\mathrm{SO}(2 \mathrm{n}+1)) \cong \wedge_{\mathbf{C}}\left(\mathrm{x}_{3}, \mathrm{x}_{7}, \ldots, \mathrm{x}_{4 \mathrm{n}-1}\right) .
\end{gathered}
$$

(c). Define a function $\Phi: \mathbf{N} \times \mathbf{N} \times \mathbf{N} \rightarrow \mathbf{Z}$ by

$$
\Phi(n, k, \varepsilon)=\sum_{i=1}^{k}(-1)^{i-1}\left(\begin{array}{c}
n \\
k-i
\end{array}\right) i^{q-1} .
$$

It then follows from [16], 17] that we have Chern character $c h: K^{*}(\mathrm{SU}(\mathrm{n}+1)) \rightarrow$ $\mathrm{H}^{*}(\mathrm{SU}(\mathrm{n}+1))$ given by

$$
\operatorname{ch}\left(\beta\left(\rho_{k}\right)\right)=\sum_{i=1}^{n} \frac{(-1)^{i}}{i !} \Phi(n+1, k, i+1) x_{2 i+1}, \forall k \geq 1,
$$

ch $: K^{*}(\mathrm{SO}(2 \mathrm{n}+1)) \rightarrow \mathrm{H}^{*}(\mathrm{SO}(2 \mathrm{n}+1))$, given by the formula

$$
\operatorname{ch}\left(\beta\left(\lambda_{k}\right)\right)=\sum_{i=1}^{n} \frac{(-1)^{i-1} 2}{(2 i-1) !} \Phi(2 n+1, k, 2 i) x_{4 i-1}(\forall k=1,2, \ldots, n-1)
$$




$$
\operatorname{ch}\left(\varepsilon_{2 n+1}\right)=\sum_{i=1}^{n} \frac{(-1)^{i-1}}{2^{n-1}(2 i-1) !} \sum_{k=1}^{n} \Phi(2 n+1, k, 2 i) x_{4 i-1} .
$$

\section{Algebraic Version of Non-Commutative Chern Character}

Let $G$ be a compact Lie group, $\mathrm{HP}_{*}\left(\mathrm{C}^{*}(\mathrm{G})\right)$ the periodic cyclic homology introduced in $\S 2$. Since $C^{*}(G)=\lim _{\longrightarrow} N \prod_{i=1}^{N} \operatorname{Mat}_{\mathrm{n}_{\mathrm{i}}}(\mathbf{C}), \operatorname{HP}_{*}\left(\mathrm{C}^{*}(\mathrm{G})\right)$ coincides with the $\operatorname{HP}_{*}\left(\mathrm{C}^{*}(\mathrm{G})\right)$ defined by J. Cuntz-D. Quillen [6].

Lemma 4.1. Let $\left\{I_{N}\right\}_{N \in \mathbf{N}}$ be the above defined collection of ideals in $C^{*}(G)$. Then

$$
K_{*}\left(C^{*}(G)\right)=\lim _{N \in \mathbf{N}} K_{*}\left(I_{N}\right)=K_{*}^{W}(\mathbf{C}(\mathbf{T})),
$$

where $\mathbf{T}$ is the fixed maximal torus of $G$.

Proof. First note that the algebraic K-theory of $C^{*}$-algebras has the stability property

$$
K_{*}\left(A \otimes M_{n}(\mathbf{C})\right) \cong K_{*}(\mathbf{C})
$$

Hence,

$$
\lim _{\longrightarrow} K_{*}\left(I_{n_{i}}\right) \cong K_{*}\left(\prod_{\lambda=\text { highest weight } \bmod \mathrm{W}} \mathbf{C}_{\lambda}\right) \cong K_{*}^{W}(\mathbf{C}(\mathbf{T}))
$$

by Pontryagin duality, where $\mathbf{C}_{\lambda}=\mathbf{C}$ are enumerated by the highest weights of the corresponding irreducible representations of $G$.

J. Cuntz and D. Quillen [6] defined the so called $X$-complexes of $\mathbf{C}$-algebras and then used some ideas of Fedosov product to define algebraic Chern characters. We now briefly recall their definitions. For a (non-commutative) associate $\mathbf{C}$-algebra $A$, consider the space of even non-commutative differential forms $\Omega^{+}(A) \cong R A$, equipped with the Fedosov product

$$
\omega_{1} \circ \omega_{2}:=\omega_{1} \omega_{2}-(-1)^{\left|\omega_{1}\right|} d \omega_{1} d \omega_{2},
$$

see [6]. Consider also the ideal $I A:=\oplus_{k \geq 1} \Omega^{2 k}(A)$. It is easy to see that $R A / I A \cong A$ and that $R A$ admits the universal property that any based linear map $\rho: A \rightarrow M$ can be uniquely extended to a derivation $D: R A \rightarrow M$. The derivations $D: R A \rightarrow M$ bijectively correspond to lifting homomorphisms from $R A$ to the semi-direct product $R A \oplus M$, which also bijectively correspond to linear map $\bar{\rho}: \bar{A}=A / \mathbf{C} \rightarrow M$ given by

$$
a \in \bar{A} \mapsto D(\rho a)
$$

From the universal property of $\Omega^{1}(R A)$, we obtain a bimodule isomorphism

$$
R A \otimes \bar{A} \otimes R A \cong \Omega^{1}(R A) .
$$

As in [6], let $\Omega^{-} A=\oplus_{k \geq 0} \Omega^{2 k+1} A$. Then we have

$$
\Omega^{-} A \cong R A \otimes \bar{A} \cong \Omega^{1}(R A)_{\#}:=\Omega^{1}(R A) /\left[\left(\Omega^{1}(R A), R A\right)\right] .
$$

J. Cuntz and D. Quillen proved 
Theorem 4.2. ([6], Theorem1): There exists an isomorphism of $\mathbf{Z} /(2)$-graded complexes

$$
\Phi: \Omega A=\Omega^{+} A \oplus \Omega^{-} A \cong R A \oplus \Omega^{1}(R A)_{\#},
$$

such that

$$
\Phi: \Omega^{+} A \cong R A
$$

is defined by

$$
\Phi\left(a_{0} d a_{1} \ldots d a_{2 n}=\rho\left(a_{1}\right) \omega\left(a_{1}, a_{2}\right) \ldots \omega\left(a_{2 n-1}, a_{2 n}\right)\right.
$$

and

$$
\begin{gathered}
\Phi: \Omega^{-} A \cong \Omega^{1}(R A)_{\#}, \\
\Phi\left(a_{0} d a_{1} \ldots d a_{2 n+1}\right)=\rho\left(a_{1}\right) \omega\left(a_{1}, a_{2}\right) \ldots \omega\left(a_{2 n-1}, a_{2 n}\right) \delta\left(a_{2 n+1}\right) .
\end{gathered}
$$

With respect to this identification, the product in $R A$ is just the Fedosov product on even differential forms and the differentials on the $X$-complex

$$
X(R A): \quad R A \cong \Omega^{+} A \rightarrow \Omega^{1}(R A)_{\#} \cong \Omega^{-} A \rightarrow R A
$$

become the operators

$$
\begin{gathered}
\beta=b-(1+\kappa) d: \Omega^{-} A \rightarrow \Omega^{+} A, \\
\delta=-N_{\kappa^{2}} b+B: \Omega^{+} A \rightarrow \Omega^{-} A,
\end{gathered}
$$

where $N_{\kappa^{2}}=\sum_{j=0}^{n-1} \kappa^{2 j}, \kappa\left(d a_{1} \ldots d a_{n}\right):=d a_{n} \ldots d a_{1}$.

Let us denote by $I A \triangleleft R A$ the ideal of even non-commutative differential forms of order $\geq 2$. By the universal property of $\Omega^{1}$

$$
\Omega^{1}(R A / I A)=\Omega^{1} R A /\left((I A) \Omega^{1} R A+\Omega^{1} R A .(I A)+d I A\right) .
$$

Since $\Omega^{1} R A=(R A) d R A=d R A .(R A)$, then $\Omega^{1} R A(I A) \cong I A \Omega^{1} R A \bmod \left[R A, \Omega^{1} R\right]$.

$$
\Omega^{1}(R A / I A)_{\#}=\Omega^{1} R A /\left(\left[R A, \Omega^{1} R A\right]+I A \cdot d R A+d I A\right) .
$$

For $I A$-adic tower $R A /(I A)^{n+1}$, we have the complex

$$
\mathcal{X}\left(R A /(I A)^{n+1}\right): \quad R A / I A^{n+1} \leftarrow \Omega^{1} R A /\left(\left[R A, \Omega^{1} R A\right]+(I A)^{n+1} d R A+d(I A)^{n+1}\right) .
$$

Define

$$
\begin{aligned}
& \mathcal{X}^{2 n+1}(R A, I A): \quad R A /(I A)^{n+1} \rightarrow \Omega^{1} R A /\left(\left[R A, \Omega^{1} R A\right]+(I A)^{n+1} d R A+d(I A)^{n+1}\right) \\
& \rightarrow R A /(I A)^{n+1}, \\
& \mathcal{X}^{2 n}(R A, I A): \quad R A /\left((I A)^{n+1}+\right. {\left.\left[R A, I A^{n}\right]\right) \rightarrow \Omega^{1} R A /\left(\left[R A, \Omega^{1} R A\right]+d(I A)^{n} d R A\right) } \\
& \rightarrow R A /\left((I A)^{n+1}+\left[R A, I A^{n}\right]\right) .
\end{aligned}
$$

One has

$$
\begin{gathered}
b\left((I A)^{n} d I A\right)=\left[(I A)^{n}, I A\right] \subset(I A)^{n+1}, \\
d(I A)^{n+1} \subset \sum_{j=0}^{n}(I A)^{j} d(I A)(I A)^{n-j} \subset(I A)^{n} d I A+\left[R A, \Omega^{1} R A\right] .
\end{gathered}
$$


and hence

$$
\begin{aligned}
& \mathcal{X}^{1}(R A, I A)=X(R A, I A), \\
& \mathcal{X}^{0}(R A, I A)=(R A / I A)_{\#} \cdot
\end{aligned}
$$

There is a sequence of maps between complexes

$$
\cdots \rightarrow X(R A / I A) \rightarrow \mathcal{X}^{2 n+1}(R A, I A) \rightarrow \mathcal{X}^{2 n}(R A, I A) \rightarrow X(R A / I A) \rightarrow \ldots
$$

We have the inverse limits

$$
\hat{X}(R A, I A):=\lim _{\longleftarrow} X\left(R A /(I A)^{n+1}\right)=\lim _{\longleftarrow} \mathcal{X}^{n}(R A, I A) .
$$

Remark that

$$
\begin{gathered}
\mathcal{X}^{q}=\Omega A / F^{q} \Omega A, \\
\hat{X}(R A / I A)=\hat{\Omega} A .
\end{gathered}
$$

We quote the second main result of J. Cuntz and D. Quillen ([6], Thm2), namely:

$$
H_{i} \hat{\mathcal{X}}(R A, I A)=\mathrm{HP}_{\mathrm{i}}(\mathrm{A})
$$

We now apply this machinery to our case. First we have the following.

\section{Lemma 4.3.}

$$
\underset{\lim _{\longrightarrow}}{H^{*}}\left(\mathrm{I}_{\mathrm{N}}\right) \cong \operatorname{HP}^{*}(\mathbf{C}(\mathbf{T}))
$$

Proof. By similar arguments as in the previous lemma 4.1. More precisely, we have

$$
\operatorname{HP}\left(\mathrm{I}_{\mathrm{n}_{\mathrm{i}}}\right)=\operatorname{HP}\left(\prod_{\lambda=\text { highest weight } \bmod \mathrm{W}} \mathbf{C}_{\lambda}\right) \cong \mathrm{HP}_{*}^{\mathrm{W}}(\mathbf{C}(\mathbf{T}))
$$

by Pontryagin duality.

Now, for each idempotent $e \in M_{n}(A)$ there is an unique element $x \in M_{n}(\widehat{R A})$. Then the element

$$
\tilde{e}:=x+\left(x-\frac{1}{2}\right) \sum_{n \geq 1} \frac{2^{n}(2 n-1) ! !}{n !}\left(x-x^{2 n}\right)^{2 n} \in M_{n}(\widehat{R A})
$$

is a lifting of $e$ to an idempotent matrix in $M_{n}(\widehat{R A})$. Then the map $[e] \mapsto \operatorname{tr}(\tilde{e})$ defines the map $K_{0}(A) \rightarrow H_{0}(X(\widehat{R A}))=\mathrm{HP}_{0}(\mathrm{~A})$. To an element $g \in \mathrm{GL}_{\mathrm{n}}(\mathrm{A})$ one associates an element $p \in \mathrm{GL}(\widehat{\mathrm{RA}})$ and to the element $g^{-1}$ an element $q \in \mathrm{GL}_{\mathrm{n}}(\widehat{\mathrm{RA}})$ then put

$$
x=1-q p, \text { and } y=1-p q .
$$

And finally, to each class $[g] \in \mathrm{GL}_{\mathrm{n}}(\mathrm{A})$ one associates

$$
\operatorname{tr}\left(g^{-1} d g\right)=\operatorname{tr}(1-x)^{-1} d(1-x)=d(\operatorname{tr}(\log (1-x)))=-\operatorname{tr} \sum_{n=0}^{\infty} x^{n} d x \in \Omega^{1}(A)_{\#} .
$$

Then $[g] \rightarrow \operatorname{tr}\left(g^{-1} d g\right)$ defines the map $K_{1}(A) \rightarrow H H_{1}(A)=H_{1}(X(\widehat{R A}))=\operatorname{HP}_{1}(\mathrm{~A})$. 
Definition 4.4. Let $\mathrm{HP}\left(\mathrm{I}_{\mathrm{n}_{\mathrm{i}}}\right)$ be the periodic cyclic cohomology defined by Cuntz-Quillen.

Then the pairing

$$
K_{*}^{a l g}\left(C^{*}(G)\right) \times \bigcup_{N} \mathrm{HP}^{*}\left(\mathrm{I}_{\mathrm{N}}\right) \rightarrow \mathbf{C}
$$

defines an algebraic non-commutative Chern character

$$
c h_{a l g}: K_{*}^{a l g}\left(C^{*}(G)\right) \rightarrow \operatorname{HP}_{*}\left(\mathrm{C}^{*}(\mathrm{G})\right),
$$

which gives us a variant of non-commutative Chern characters with values in HP-groups.

We close this section with an algebraic analogue of theorem 3.2.

Theorem 4.5. Let $G$ be a compact Lie group and $\mathbf{T}$ a fixed maximal compact torus of $G$. Then in the notations of 4.3, the Chern character

$$
c h_{\text {alg }}: K_{*}\left(C^{*}(G)\right) \rightarrow \mathrm{HP}_{*}\left(\mathrm{C}^{*}(\mathrm{G})\right)
$$

is an isomorphism, which can be identified with the classical Chern character

$$
c h: K_{*}^{W}(\mathbf{C}(\mathbf{T})) \rightarrow \mathrm{HP}_{*}^{\mathrm{W}}(\mathbf{C}(\mathbf{T}))
$$

which is also an isomorphism.

Proof. First note that

$$
\begin{aligned}
& K_{*}\left(C^{*}(G)\right) \cong K_{*}\left(\varliminf_{i=1} \prod_{i=1}^{N} \operatorname{Mat}_{n_{\mathrm{i}}}(\mathbf{C})\right)=\lim _{\longrightarrow} \mathrm{K}_{*}\left(\prod_{\mathrm{i}=1}^{\mathrm{N}} \operatorname{Mat}_{\mathrm{n}_{\mathrm{i}}}(\mathbf{C})\right) \cong \\
& \cong K_{*}\left(\prod \quad \mathbf{C}_{\lambda}\right)=K_{*}(\mathbf{C}(\mathbf{T})) \text {. } \\
& \lambda=\text { highest weight } \bmod \mathrm{W}
\end{aligned}
$$

Next we have

$$
\begin{aligned}
& \mathrm{HP}_{*}\left(\mathrm{C}^{*}(\mathrm{G})\right) \cong \mathrm{HP}_{*}\left(\lim _{\mathrm{i}=1} \prod_{\mathrm{N}=1}^{\mathrm{N}} \operatorname{Mat}_{\mathrm{n}_{\mathrm{i}}}(\mathbf{C})\right)=\underline{\lim _{\longrightarrow}} \operatorname{HP}_{*}\left(\prod_{\mathrm{i}=1}^{\mathrm{N}} \operatorname{Mat}_{\mathrm{n}_{\mathrm{i}}}(\mathbf{C})\right) \\
& \cong \mathrm{HP}\left(\quad \mathbf{C}_{\lambda}\right)=\mathrm{HP}_{*}^{\mathrm{W}}(\mathbf{C}(\mathbf{T})) \text {. }
\end{aligned}
$$

Furthermore, by a result of Cuntz-Quillen for the commutative $\mathbf{C}$-algebra $A$, we have a canonical isomorphism from periodic cyclic homology to the $\mathbf{Z} /(2)$-graded de Rham homology which is an isomorphism when $A$ is smooth. Hence

$$
K_{*}^{W}(\mathbf{C}(\mathbf{T})) \cong \mathrm{HP}_{*}^{\mathrm{W}}(\mathbf{C}(\mathbf{T})) .
$$

Now we have a commutative diagram

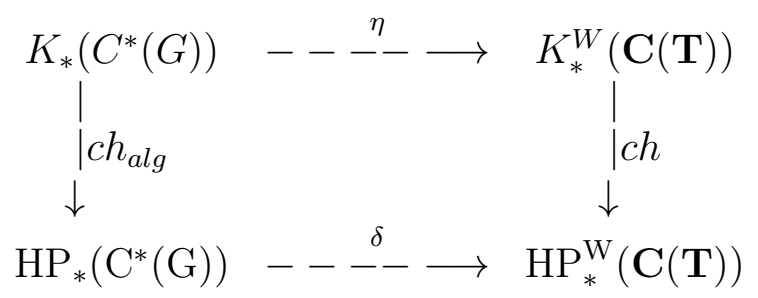

where $\eta, \delta, c h$ are isomorphisms. Hence, $c h_{a l g}=\delta^{-1}(c h) \eta$ is an isomorphism. 


\section{ACKNowledGments}

This work was completed during the stay of the first author as a visiting mathematician at the International Centre for Theoretical Physics, Trieste, Italy. He would like to thank ICTP for the hospitality, without which this work would not have been possible.

This work is supported in part by the International Centre for Theoretical Physics, Trieste, Italy and the National Foundation for Research in Natural Sciences of Vietnam.

\section{REFERENCES}

[1] N. Berline, E. Getzler and M. Vergne, Heat Kernels and Dirac operators, Grundlehren der mathematischen Wissenschaften, Corrected 2nd Printing 1996, No. 298, Springer-Verlag,1992.

[2] A. Borel, Sur la cohomologie des espaces fibrés principaux et des espaces homogènes de groupes de Lie compacts, Annals of Math., 57(1953), 115-207.

[3] A. Connes, Non commutative Geometry, Academic Press, 1994.

[4] A. Connes, Entire cyclic cohomology of Banach algebras and characters of $\theta$-summable Fredholm modules, K-theory, 1(1988), 519-548.

[5] J. Cuntz, A survey of some aspects of non-commutative geometry, Mathematisches Inst. Uni. Heidelberg, 35(1992), pp.1-29.

[6] J. Cuntz and D. Quillen, The $X$ complex of the universal extensions, Preprint Math. Inst. Uni. Heidelberg, 1993.

[7] D. N. Diep and N. V. Thu, Homotopy invariance of entire current cyclic homology, Vietnam J. of Math., 25(1997), No 1, 211-228.

[8] D. N. Diep and N. V. Thu, Entire homology of non-commutative de Rham currents, ICTP, IC/96/214, 1996, 23pp; to appear in Publication of CFCA, Hanoi-City Vietnam National University, 1997.

[9] F. Feng and B. Tsygan, Hochschild and cyclic homology of quantum groups, Commun. Math. Phys. 140(1991), 481-521.

[10] L. Hodgkin, On the K-theory of Lie groups, Topology, 6(1967), 1-36.

[11] M. Khalkhali, On the entire cyclic cohomology of Banach algebras: I. Morita invariance, Mathematisches Inst. Uni. Heidelberg, 54(1992), pp 24.

[12] M. Khalkhali, On the entire cyclic cohomology of Banach algebras: II. Homotopy invariance, Mathematisches Inst. Uni. Heidelberg, 55(1992), pp 18.

[13] A. A. Kirillov, Elements of the Theory of representations, Springer-Verlag, Berlin-Heidelberg-New York, 1975.

[14] N. V. Thu, Morita invariance of entire current cyclic homology, Vietnam J. Math. (to appear).

[15] N. V. Thu, Exactness of entire current cyclic homology, Acta Math. Vietnamica (to appear).

[16] T. Watanabe, On the Chern characters of symmetric spaces related to $S U(n)$, J. Math. Kyoto Univ. 34(1994), 149-169.

[17] T. Watanabe, Chern characters on compact Lie groups of low rank, Osaka J. Math. 22(1985), 463488. 